\title{
Aperfeiçoamentos na triangularização do Marching Cubes para geração de menos triângulos degenerados
}

\author{
Thiago F. Leal, \\ PPGEM - UERJ, 20550-900, Rio de Janeiro, RJ \\ IFRJ, 26600-000, Paracambi, RJ \\ E-mail: thiagofranco@ime.uerj.br
}

Cassia I. G. Silva

IC - UFF, 24210-240, Niterói, RJ

IFRJ, 26600-000, Paracambi, RJ

E-mail: cassiaisac@ic.uff.br

\author{
Aruquia B. M. Peixoto, \\ DEPB-NI, CEFET/RJ \\ 26041-271, Nova Iguaçu, RJ \\ E-mail: aruquia@gmail.com
}

\author{
Marcelo de A. Dreux, \\ DEM - Pontifícia Universidade Católica - RJ \\ 22453-900, Rio de Janeiro, RJ \\ E-mail: dreux@puc-rio.br
}

\author{
Carlos A. de Moura, \\ IME - Universidade do Estado do Rio de Janeiro \\ 20550-900, Rio de Janeiro, RJ \\ E-mail: demoura@ime.uerj.br
}

\begin{abstract}
Resumo: $O$ Marching Cubes é um algoritmo utilizado na poligonalização de superfícies implícitas, criando uma malha de triângulos que modela um volume específico. Durante este processo, entretanto, pode ocorrer a formação de triângulos degenerados, prejudiciais à aplicação adequada de métodos numéricos sobre esta malha. Visando inibir essa impropriedade, o presente trabalho propõe alterações no processo de triangularização do Marching Cubes, aplicando estratégias como mudança na conexão de vértices e inserção de arestas. São calculadas as medidas dos ângulos dos polígonos gerados e, estando estes menores do que um parâmetro pré-estabelecido, o algoritmo efetua a correção apropriada a fim de melhorar a qualidade dos triângulos gerados.
\end{abstract}

\section{Marching Cubes}

O Marching Cubes (MC) é um algoritmo que cria uma representação tridimensional de um volume. A superfície é poligonalizada e são usados os valores de uma função implícita como base para determinar a posição dos pontos da superfície e formatar o modelo.

Sua primeira aplicação foi na representação de imagens médicas, onde a partir de uma série de imagens bidimensionais era possível reconstruir computacionalmente um molde gráfico da anatomia do paciente. Isso tornou mais fácil a realização de exames de imagem e visualização de fraturas e traumatismos ósseos.

\subsection{Funcionamento do MC}

Inicialmente, o algoritmo gera um cubo no qual a superfície de interesse possa estar totalmente contida. Este cubo será subdividido em novos cubos (voxels) suficientemente pequenos e de mesmo tamanho, fatiando uniformemente seu volume interno e, consequentemente, a superfície ali contida.

Realizada esta etapa, o algoritmo cria um "cubo lógico" que irá percorrer cada célula da grade, capturando os pontos de interseção da superfície com suas arestas. A conexão dos pontos forma um polígono e seus vértices são conectados três a três, dando formato à triangularização naquela célula. O MC então "marcha" para a próxima célula do reticulado uniforme e segue nova análise e construção do modelo. 
O MC utiliza uma tabela específica com 14 padrões possíveis para as triangularizações, representados pela ilustração abaixo.
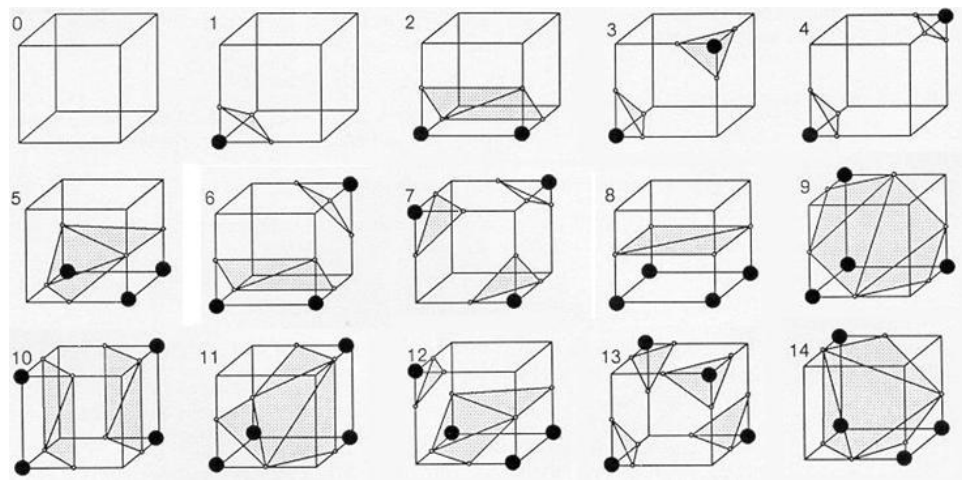

Figura 1: Tabela de triangularização do Marching Cubes. Extraído de [3].

Ao fim dessa operação, isto é, após varrer toda a grade e realizar as triangularizações necessárias, obtém-se o molde gráfico do objeto, construído a partir de uma malha de triângulos.

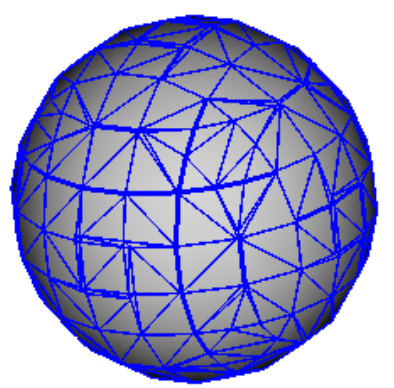

Figura 2: Poligonalização de uma esfera.

\subsection{Triângulos degenerados}

A utilização do Marching Cubes, entretanto, pode ocasionar pequenos problemas devido a limitações do algoritmo. É comum a geração de triângulos degenerados, com pelo menos um de seus ângulos internos muito pequeno. Quando o propósito da poligonalização é obter uma malha onde serão aplicadas técnicas numéricas, os triângulos degenerados podem influenciar o tratamento do problema.
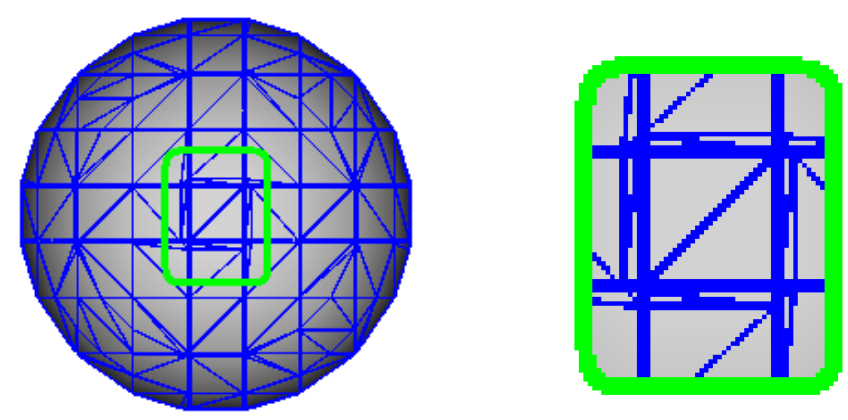

Figura 3: Triângulos degenerados. 


\section{Modificações}

A proposta envolve a realização de testes e aplicação de estratégias a casos específicos durante o processo de triangularização do algoritmo. Nos casos onde têm-se triângulos isolados, nada é feito. Isto ocorre, por exemplo, nos casos básicos 1, 3, 4, 6, 7, 12 e 13 da Figura 1.
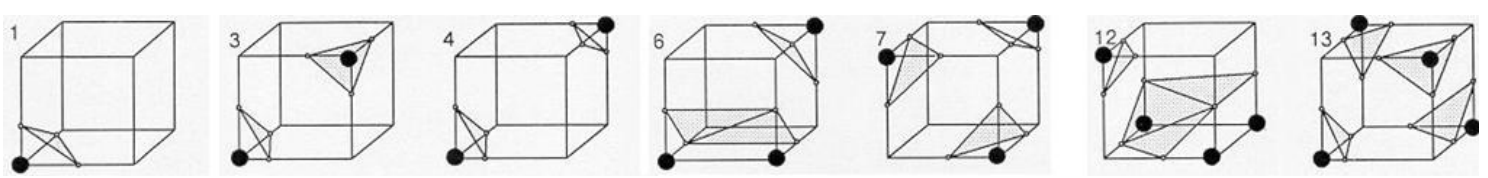

Figura 4: Casos 1, 3, 4, 6, 7, 12 e 13 da tabela do MC que apresentam triângulos isolados.

Vale notar que nos casos 6 e 12 existem triângulos isolados, mas também regiões formadas por triângulos conectados entre si. Como previsto, nada será feito com o triângulo isolado. Os demais, entretanto, formam um polígono, cujo bordo deve ser considerado para gerar a triangularização. Neste, são determinadas as medidas de cada lado e ângulo interno. Se algum ângulo for menor que o parâmetro predeterminado, o MC gera um triângulo simplesmente ligando os vértices adjacentes ao que apresenta ângulo pequeno. Isso evita utilizar esse vértice numa nova triangularização, o que conduziria à divisão de um ângulo que já apresenta medida indesejada.

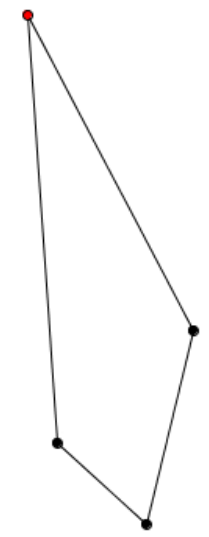

(a)

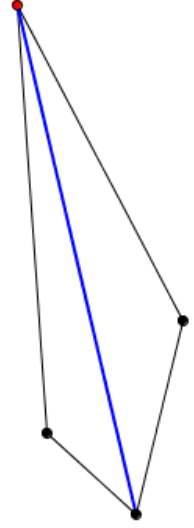

(b)

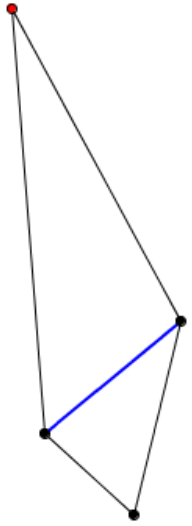

(c)

Figura 5: (a) Polígono com ângulo (suficientemente) pequeno no vértice vermelho; (b) Possível triangularização, com divisão do ângulo pequeno em dois menores; (c) Conexão dos vértices adjacentes, não havendo divisão do ângulo do vértice destacado.

O passo seguinte à conexão de vértices descrita, que isola os vértices cujos ângulos têm medida suficientemente pequena, utiliza as medidas dos lados do polígono, para polígonos com quatro ou mais vértices. É calculada a razão entre o maior e o menor dos lados do polígono. Se o valor desta razão for maior do que o parâmetro escolhido, um novo vértice é inserido no interior da célula e todos são conectados a este, como em [2]. Porém, na referência citada, o vértice é inserido exatamente no meio do polígono, enquanto neste trabalho a inserção é ponderada e feita em posição mais conveniente.

Este novo vértice deve ser alocado mais próximo do lado de menor medida, a fim de que o triângulo formado não tenha uma base muito pequena quando comparado com os outros dois lados, o que poderia resultar em sua degeneração. A aproximação do novo vértice ao menor lado ocorre naturalmente quando as medidas dos lados são ponderadas por um fator em função do 
valor da razão entre as medidas dos lados. Isto motiva a localização adequada do novo vértice de acordo com o objetivo.

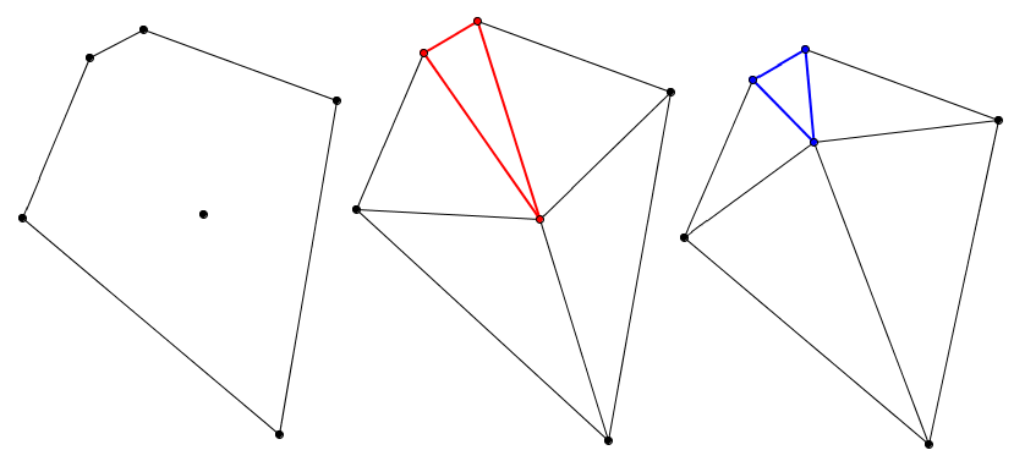

Figura 6: A inserção do novo vértice, nesta proposta, deve ser feita mais próxima do menor lado do polígono, evitando a formação de um triângulo degenerado.

Se esses procedimentos não forem necessários, isto é, se a razão entre o maior e o menor lado for inferior ao valor do parâmetro, é feita a conexão dos vértices do polígono. Essa conexão é efetuada unindo o vértice relativo ao maior ângulo do polígono a um dos dois vértices que está a duas arestas de distância. Destes dois vértices é escolhido aquele que tiver maior ângulo, formando assim um triângulo. Este processo é repetido no polígono restante até que a triangularização esteja completa. Uma vez que estamos dividindo os maiores ângulos, esta deve ser a melhor situação possível para a geração de triângulos neste voxel.

\section{Resultados e discussão}

A geração de malhas com o Marching Cubes, utilizando as melhorias aqui propostas e ajuste conveniente dos parâmetros, possibilitou observar a redução do número de triângulos degenerados na malha.

Foram feitos testes em superfícies diferentes, onde estas foram poligonalizadas segundo a tabela original do $\mathrm{MC}$ e, posteriormente, comparadas com a poligonalização resultante das alterações sugeridas. Os resultados são exibidos a seguir.
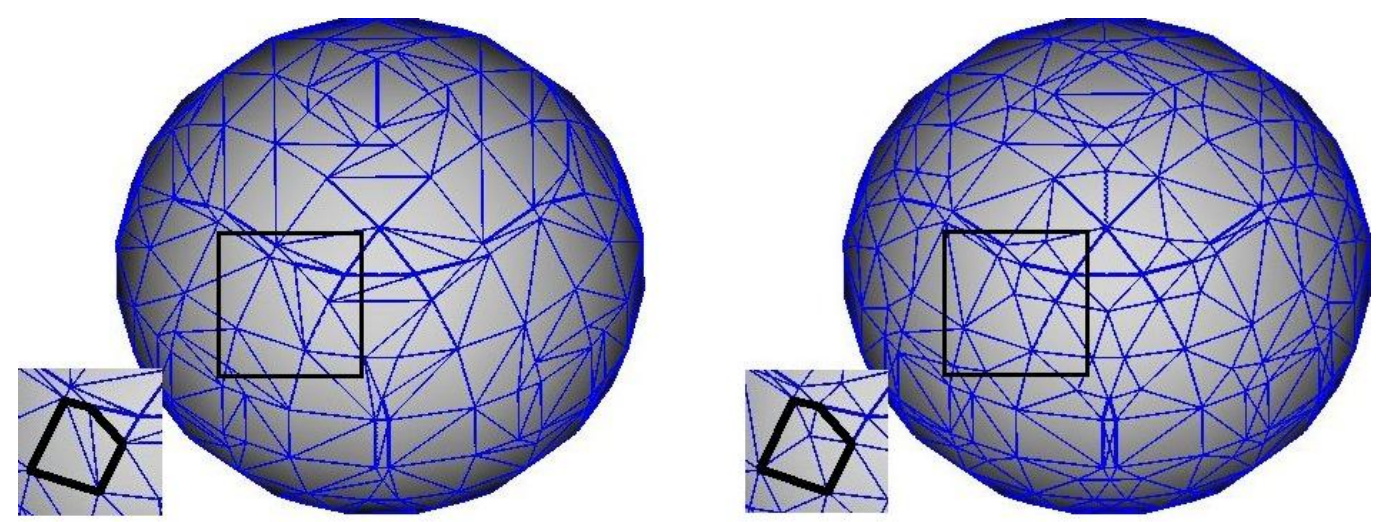

Figura 7: Esfera. A malha original gerada pelo MC está à esquerda. À direita a malha obtida com o método proposto. Em detalhe, um exemplo de região onde houve sensível melhora na qualidade dos triângulos. 
Outras superfícies:
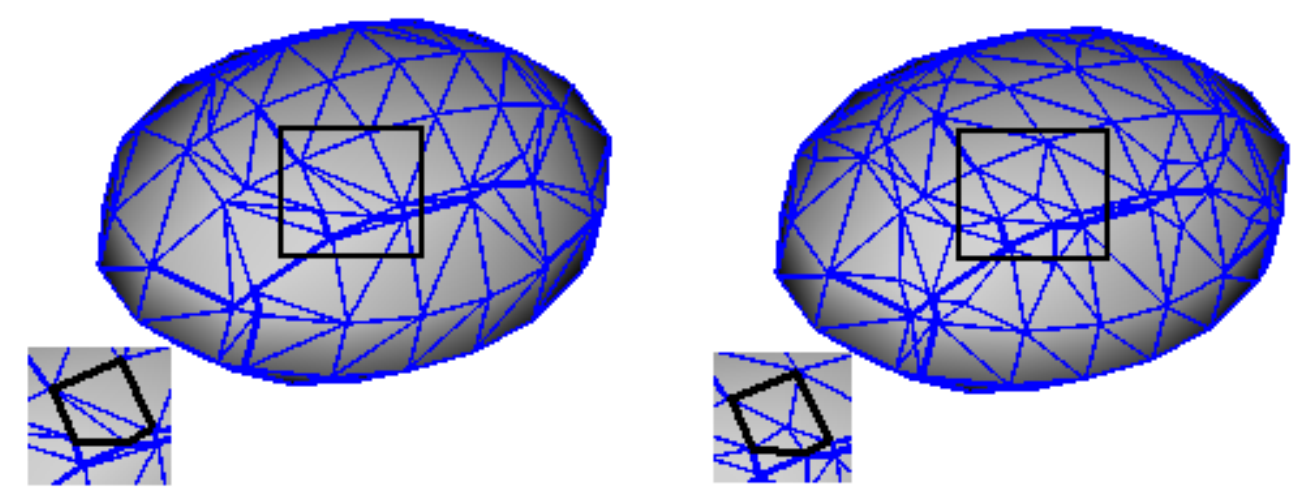

Figura 8: Elipsoide.
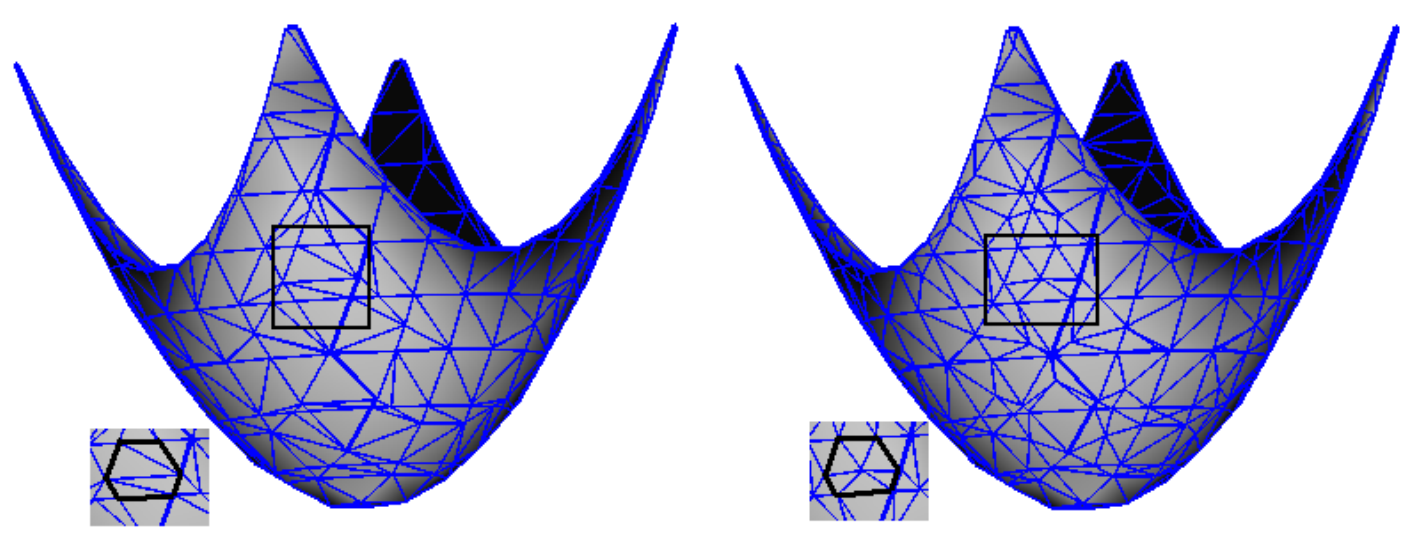

Figura 9: Paraboloide.
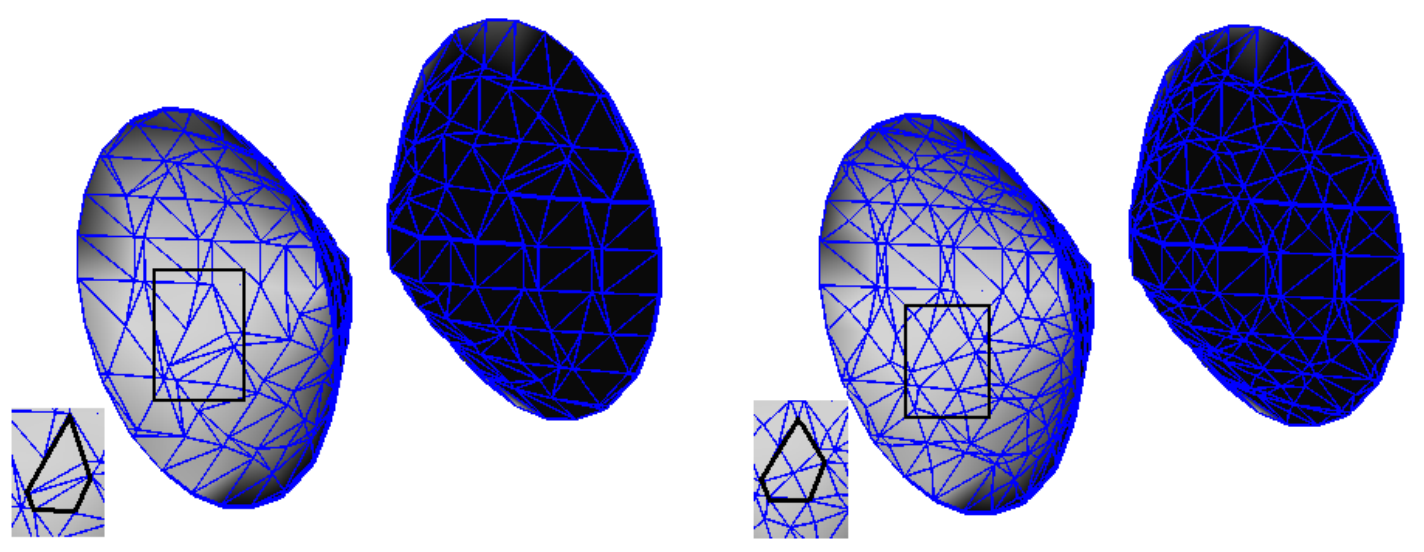

Figura 10: Hiperboloide de duas folhas. 

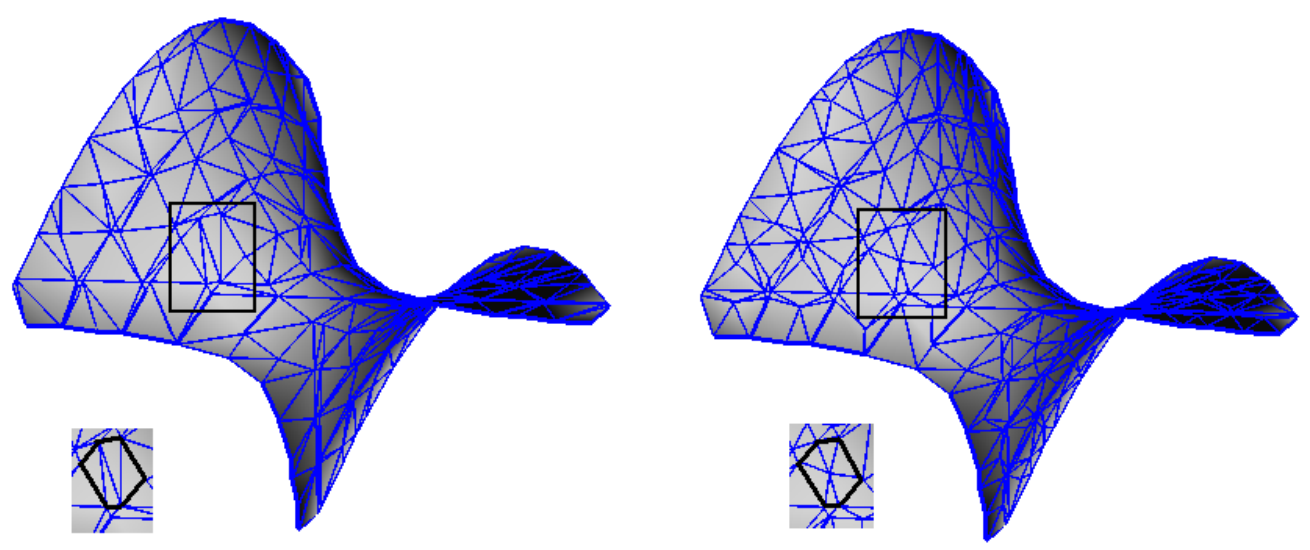

Figura 11: Paraboloide hiperbólico (sela).

Apesar das alterações realizadas, ainda pode haver triângulos com ângulos abaixo do valor desejado. Isto ocorre devido a duas decisões tomadas ao longo do procedimento:

a. Quando o resultado da poligonalização em um voxel é um único triângulo, estes não são modificados, permanecendo como foram originalmente gerados e eventualmente podendo apresentar ângulos pequenos;

b. Antes da adição de um novo vértice à célula, se houver um ou mais ângulos muito pequenos, estes dão origem a triângulos por meio da conexão de vértices adjacentes, evitando subdividilos.

Novos ajustes podem ser realizados com técnicas de pós-processamento, visando reduzir ainda mais a quantidade de triângulos degenerados. A pesquisa apresenta, portanto, possibilidades interessantes de extensão, as quais já estão em andamento. Um resumo deste trabalho foi apresentado no 11th US National Congress on Computational Mechanics [3].

\section{Referências}

[1] CIGNONI, P.; MONTANI, C.; PUPPO, E.; SCOPIGNO, R., "Multiresolution Representation and Visualization of Volume Data". Technical Report C97-05, CNUCE - C.N.R., Pisa, Italy, January 1997.

[2] DIETRICH, C. A.; SCHEIDEGGRE, C. E.; COMBA, J. L. D.; NEDEL, L. P.; SILVA, C. T., "Marching cubes without skinny triangles," Computing in Science and Engg., vol. 11, no. 2, pp. 82-87, 2009.

[3] LEAL, T. F. ; PEIXOTO, A. B. M. ; DREUX, M. de A. ; MOURA, C A de . Generation of Triangular Meshes through Vertices Connection Modification of the Marching Cubes Algorithm. 11th USNCCM - US National Congress on Computational Mechanics, Minneapolis, EUA, 2011.

[4] LORENSEN, W. E.; CLINE, H. E., "Marching cubes: A high resolution 3D surface construction algorithm", Proceedings of the 14th annual conference on Computer graphics and interactive techniques, pp.163-169, August 1987.

[5] SHEWCHUK, J., "What is a Good Linear element? Interpolation, Conditioning, and Quality measures", 11th International Meshing Roundtable, pp. 115-126, Sandia National Laboratories, 2002. 